\title{
Ashes2Art: Mitigation Strategies for Short-and Long-term Distress in Emergency Services Personnel During COVID-19
}

\author{
Holly C. Matto ${ }^{1} \cdot$ Kathy Sullivan ${ }^{2}$ \\ Accepted: 5 February 2021 / Published online: 25 February 2021 \\ (c) The Author(s), under exclusive licence to Springer Science+Business Media, LLC part of Springer Nature 2021
}

\begin{abstract}
Ashes2Art, a nonprofit organization working with fire fighters and first responders since 2017, promotes creativity to counter balance the exposure to extreme loss and trauma. Operating under the Northern Virginia Emergency Medical Services Council, Ashes2Art provides art supplies, art classes, and a creative community of support to mitigate the deleterious effects the stress of the job can take on fire fighters and first responders' health and mental health. During the COVID-19 pandemic, Ashes2Art has seen an increased demand for art supplies and the, now online, creative arts classes and self-care strategies. Currently, approximately 100 emergency services personnel and family members are actively participating in these initiatives during this crisis. Managing the cumulative emotional load these first responders and their families experience is paramount to COVID-19 recovery efforts and post-pandemic operations. Helping first responders and their families manage the shortand long-term emotional toll from the work they do in responding to the COVID-19 crisis is paramount to the United States' successful recovery back to a well-functioning post-pandemic society. This paper suggests that enhancing well-being through mindfulness-focused creative arts engagement might be one effective tool to be included as part of routine self-care protocols for first responders and their families.
\end{abstract}

Keywords Arts techniques · Mindfulness · First responders $\cdot$ Resiliency

\section{Introduction}

Our country is in the midst of a public health pandemic while simultaneously experiencing widespread economic, social justice and political crises, all of which are taking a significant biopsychosocial toll on many individuals, families and communities. Indeed, studies show that biological, man-made and natural disasters significantly impact wellbeing, increasing anxiety, depression and other mental health symptomology (Fergusson et al. 2014). Furthermore, studies show that cumulative exposure to disasters increases risk for both poor mental health (e.g., anxiety, depression, posttraumatic stress disorder) and poor physical health (Lowe et al.

Holly C. Matto

hmatto@gmu.edu

Kathy Sullivan

kathy@ kathysullivanart.com

1 Department of Social Work, George Mason University, Peterson Family Health Sciences Hall, Fairfax, VA, USA

2 Ashes2Art Program, Fairfax, VA, USA
2019). Studies show stress in firefighters increase on-duty, as compared to off-duty, and increase over time on shift; while, heterogeneity in off-duty stress may be accounted for by different family-life experiences and available supports (Courtney et al. 2020). Specifically, firefighters and other first responders experience notable burdens during this pandemic (Miller 2020). For example, emergency services personnel, including firefighters, have high risk of occupationrelated disease or infection exposure (Baker et al. 2020); thus, COVID-19 exposure risk is of natural heightened concern for firefighters and their families.

Pre-disaster racial and socio-economic disparities influence post-disaster resiliency outcomes (Mayer 2019); and, COVID-19 data show alarming racial and socio-economic disparities (Roland 2020). For example, predominantly African-American counties are three times as likely as predominantly white counties to contract COVID-19 and six times as likely to die from the disease (Yancy 2020). The country's long-standing structural inequities and adverse social determinants of health, such as housing and food insecurity exposed by this pandemic, and the civil protests against racial injustice and systemic inequities, add layers 
of uncertainty and emotional intensity to the existing public health crisis for both those who seek help in the pandemic and those working on the pandemic frontlines (Roland 2020).

Thus, there is significant concern for emergency services personnel during this pandemic, with increasing role strain and disruptions to their work-family life. Studies show frontline workers have important concerns about their professional role during and after disaster relief efforts. For example, workers during war experience permeable boundaries between their professional work life and family life, exhibiting heightened worry about their families' safety (Segal-Engelchin et al. 2020). Chronic trauma exposure from man-made or natural disasters, especially experienced early in one's career, can have long-term impact on physical, mental and interpersonal functioning (Bracken-Scully \& McGilloway 2016). Emergency services personnel, during the COVID-19 pandemic, experience similar worry, and are uniquely affected by mandatory quarantine after traumatic exposure. In line with prior research, first responders worry for colleagues and, when isolated from family, worry about their families contracting the virus. As part of their work, they confront acute grief and loss often without timely mechanisms in place to process and appropriately grieve. Concern about family safety as well as one's health, along with the uncertainty, social isolation and exposure to loss and trauma, are all significant enduring challenges confronting frontline workers and first responders during the COVID-19 pandemic.

Table 1 illustrates the extreme polarities experienced at the convergence of these crises. Living at the extremes across these domains, while simultaneously needing to isolate from friends and family, poses a serious risk to wellbeing. Extended social isolation, whether through formal workplace quarantine protocols, Governors' stay-at-home orders, or self-imposed isolation due to fear, has created gaps in meaningful human connection. For example, one-fifth of

Table 1 Pandemic polarities

\begin{tabular}{ll}
\hline Crisis & Growth \\
\hline Vulnerability & Safety \\
Uncertainty & Predictability \\
Powerlessness & Control \\
Isolation & Connection \\
Loneliness & Unity \\
Fear & Assurance \\
Anxiety & Calm \\
Insecurity & Confidence \\
Exhaustion & Energy \\
Tension & Release \\
Illness & Health \\
Destruction & Creation \\
\hline
\end{tabular}

adults in the United States, before the pandemic, experienced loneliness or social isolation, and those who experience loneliness are more likely to have physical and mental health problems (DiJulio et al. 2018). Heightened anxiety experienced due to social distancing protocols in both work and family domains can create discontinuity in these supportive relationships (Walsh 2020). For firefighters, this has been particularly challenging. These cumulative stressors about family health and well-being, specific to this pandemic, in addition to the routine cumulative stress exposures inherent in emergency services personnel's daily work, create a condition where the risk to mental and physical health is high for this population, with further research needed to design tailored interventions. For example, Courtney, Lipsey, Braun, Henry, Nelson and Li (2020) state: "Future research examining how firefighters' lives outside of their jobs relate to stress and tiredness could aid researchers in identifying potential interventions for reducing firefighters' mean stress and stress variability" (p. 868).

Self-care strategies can be effective in mitigating these outcomes. Organizational implementation of self-care strategies can decrease burnout and enhance well-being. For example, a mindfulness-yoga intervention decreased stress and anxiety and increased resilience in healthcare workers (Ofei-Dodoo et al. 2020); and, informal debriefings led to increased compassion satisfaction and decreased burnout in fire and rescue personnel (Miller \& Unruh 2019). Although many organizations are increasingly recognizing the importance of self-care for emergency services personnel, the format is typically didactic and information-driven, rather than experiential and oriented around skill development. The self-care needs of emergency services personnel and their families, amplified by the current pandemic, require more than just information distribution, to also include experiential opportunities to learn valuable self-care techniques. Thus, providing a safe space for community connection, selfexpression through artistic skill development, and emotional communication from a strengths-based framework, tenets of social work practice, are implemented in Ashes2Art classes. Ashes2Art is not a structured therapeutic intervention with treatment goals; rather, it offers open, experiential creative arts classes that focus on self-care and promote wellness among emergency services personnel and their families.

\section{Coping Well in Uncertain Times: Finding the Mindful Middle with the Creative Arts}

Mindfulness-based stress reduction is evidence-based with demonstrated effectiveness in improving mental health in healthcare professionals (Shapiro et al. 2005). For example, in a sample of 121 firefighters, four-weeks of mindfulness, as compared to relaxation training or no training control, increased resiliency (Denkova et al. 2020). Mindfulness 
techniques have been integrated with art therapy to enhance self-care and reduce burnout in health care workers working in end-of-life care, with the intent to provide a community of support (Ho et al. 2019). Ho et al (2019) used a structured intervention that included mandalas for meaning-making around topics of self-care and professional purpose.

Social workers routinely use mindfulness training, focused on developing awareness, attention, and cognitive control in response to sensory input, in their health promotion work (Garland et al. 2015). Social workers skilled in mindfulness techniques could integrate that training with the physical engagement of the creative arts to offer a holistic mind-body self-care experience for clients in crisis. Finding personal sources of meaning during crisis is a significant predictor of successful functioning (Masterson-Duva et al. 2020), and mindfulness-based creative arts that promotes attention to present experiences with active engagement strategies designed to reduce reactivity to those experiences, stands to increase resiliency (Beerse et al. 2020; Ho et al. 2019). Feeling connected to a community-based arts group can facilitate wellbeing by building trusting relationships with other group members, an experience called "collective bonding", and by developing a sense of purpose and competence through learning and enacting new skills (Williams et al. 2019). Studies show that even a short-term (45 min) unstructured art-making session can decrease cortisol levels and stress and increase joy (Kaimal et al. 2016), suggesting even making materials available with minimal instruction may allow for meaningful creative engagement in a process that brings about enhanced well-being. A scoping review by the World Health Organization (WHO) summarized the impact of the creative arts on preventing and treating both physical and mental illness and in promoting physical, social and mental well-being (2019). For example, drawing offers opportunities for self-regulation and can facilitate meaningmaking out of challenging experiences:

Collages and drawing classes have been found to improve inter-professional working and to help identify team issues for doctors and nurses $(462,463)$, while art appreciation classes have been found to improve tolerance with ambiguity (464). Arts activities can reduce exhaustion and death anxiety and increase emotional awareness in those working in end-of-life care (465). (Fancourt \& Finn 2019, p. 28).

Segal-Engelchin, Achdut, Huss and Sarid (2020) described an arts directive used to help workers cope during war-time crisis. Participants were asked to draw a stressor, draw coping resources, and then to create an integrated drawing that incorporated the stressor and coping resource, which they found facilitated stress reduction, particularly when the size of the stressor was minimized in the new integrated drawing. Prior research has shown that transforming a stressful image can bring healing and growth and decrease stress (Huss \& Sarid 2014), perhaps because art-making experiences allow for safe distancing from raw feelings, while still allowing the artist to remain engaged with the emotional content embodied in the creative work (Mastandreal et al. 2019). The critical and creative thinking skills gained from direct participation in arts programming and the safe, reflective and open social space it creates may facilitate both intra- and interpersonal growth.

Art-making invites exploration of the inherent opposites and extremes that reside within the same contained space, offering the viewer the opportunity to expand ideas and grow in flexibility from initial interpretation through sustained engagement with the piece (Hass-Cohen \& Findlay 2015). Art-making allows artists and viewers into a dynamic and interactive relationship with the artwork in a negotiated space where the ambiguity of dualities can be addressed though interaction with the artwork (Barberian et al. 2019). In this way, art-making can broaden perspective, with the power to reveal new understanding of the current pandemic polarities (see Table 1) - art-making has the power to reduce these gaps across polarity extremes in essential ways. For example, themes of exhaustion and energy; fear and reassurance; tension and release; vulnerability and safety can be explored. Art-making encourages expression, control, hands-on support, and making creative connections within a safe and bounded visual space. Active engagement with the art materials, expression through the art process, and processing of the art product, can foster movement on the crisis-to-growth continuum towards energy, release, calm, and connection (Hass-Cohen \& Findlay 2015).

\section{Ashes 2 Art}

Ashes2Art, a nonprofit organization working with fire fighters and first responders since 2017, promotes creativity to counter balance the exposure to extreme loss and trauma, and to process post-traumatic stress. Operating under the Northern Virginia Emergency Medical Services Council, Ashes2Art provides art supplies, art classes, and a creative community of support to mitigate the deleterious effects the stress of the job can take on fire fighters and first responders' health and mental health. Ashes2Art does not provide therapy, but offers a creative arts space (arts instruction and art supplies) to teach fine arts techniques and build strong relationships within the emergency services personnel community. Individuals in need of a formal therapist-led psychotherapy group would be encouraged to seek that outside of their participation in Ashes2Art. The art classes are led by a trained artist, who also has a clinical background and degree, who is able to skillfully gauge the cognitive and emotional climate of the classes and redirect the instructional material 
according to the needs of the group. The focus is on teaching art skills and facilitating open-ended, generative creative solutions.

In some classes, responders were asked to use household items found at the stations or at the responder family homes to create their artwork, leveraging their creative resiliency. Giving an initial "partial directive" is important because instructions that are too prescriptive can narrow the window for creative engagement, while partial structure is still necessary to allow participants to get started in the artmaking - as the facilitator suggests to participants in the following phrase: "You get to mess up here!" - whereas, mistakes in their profession can mean the loss of life. The facilitator must be perceived as authentic and genuine in nature to gain the trust of this responder population - attention to the interpersonal relationship with responders is critical to the successful engagement of class participants. Art classes give participants a safe space to talk, connect and creatively problem-solve, as well as provide an intermediary platform to facilitate bonding that extends beyond the class itself. Many participants continue their art projects with family even after the class is over, which is representative of successful self-care and connection.

Firefighters and other emergency services personnel can maintain continuity in social connection through art-making. During the COVID-19 pandemic, Ashes2Art has seen an increased demand for art supplies and the, now online, creative arts classes. Approximately 100 emergency services personnel, including emergency room nurses, dispatchers, Transportation Security Administration (TSA), airport authority, paramedics, firefighters, law enforcement, search and rescue and their families have been served by the program in the greater Washington, DC region since the start of the COVID-19 pandemic. This novel coordinated effort, among typically disparate emergency services branches, aims to continue building an inclusive community of emergency services personnel, where the creative arts can help them connect and network in ways that strengthen support and improve well-being. Peer support is a key resource to Ashes2Art programming. Peer support offers emotional and instrumental support to participants, with credibility and trust coming from their shared professional career experiences. Peer support specialists participate in a training curriculum and are the liaisons to each of the participating fire stations for Ashes2Art initiatives. Fairfax County Fire and Rescue peer support personnel have been pivotal in implementing the Ashes2Art platform.

Managing the cumulative exposure to acute stress over a typical 30-year fire fighter career, requires a repertoire of effective coping strategies and adaptive tools. Sensory experience associated with traumatic exposure - the smells, sights, taste, touch and sounds - can reside within a person, embedded in brain and body, for long duration post-exposure (Hass-Cohen \& Findlay 2015). Art technique classes can be useful after experiencing difficult responder calls, as art classes require utilization of the whole brain, allowing for the titration of emotions and cognitive control, as opposed to relying on compartmentalization of the experience - "...the use of words relates to cognitive rather than visceral reactions, which allows for emotional distancing and mastery" (Barberian et al. 2019, p. 355). Compartmentalization can be a necessary skill to manage in the short-term; however, it can get in the way of being able to integrate new experiences in the longterm (Bracken-Scully \& McGilloway 2016). Art classes invite the artist into learning experiences where they can tolerate a range of feelings and come to realize they can still function well afterwards.

The mandala can be a vital regulation and wellness tool (Henderson et al. 2007). The mandala, a technique where artists create their work inside either a pre-designed controlled space, which sometimes uses the Zentanlge method or a blank circle, has a strong research base as a technique that improves well-being (Campenni \& Hartman 2020). Mandalas have been used to evoke safety as a response to trauma exposure in frontline workers (Grebe 2019), and randomized controlled trials show mandalas decrease anxiety and negative mood (Babouchkina \& Robbins 2015; Carsley \& Heath 2019; Lee 2018) and enhance state body mindfulness (Campenni \& Hartman 2020). The mandala principles of "center and periphery", acknowledge impermanence through awareness of the present and the skill of 'letting go': "The way to overcome the apparent contradiction between letting go and helping others is to cultivate a view that fuses compassion and wisdom" (Van Gordon et al. 2017, p. 1722). Mandala work enhances observational skills through sustained attention, helping the artist sit well with an image, being present for the moment. Sitting well with an image through sustained observation allows one to release attachment gently and to let go once completed - without judgment. Whitaker (2016) suggests: "Observation is the crux of discernment. Wanting to skip past observation to judgement is a form of racing to the end instead of staying in the weeds. Art is already intimately linked to observation" (p. 79).

\section{Case examples}

Three Ashes2Art examples, the Mandala Challenge, a Stress Ball Activity, and the Virtual Family Summer Arts Camp, are described, illustrating how active engagement safely emerges during an art-making experience and can, over time, help to facilitate a move from crisis toward growth. 


\section{The "Mandala Challenge" and Workbook}

A "Mandala Challenge" was offered at fire stations throughout Fairfax County. Peer support and Ashes2Art joined together to provide the necessary art supplies and instructional materials, delivering art supplies to all of the stations within their three shifts. A video contained the project instructions and announced there would be an accompanying raffle for the stations, with the winning station receiving a gift certificate and a donation from Ashes2Art to the Firefighters Fund. Once stations started the challenge, others readily joined. Some firefighters brought the supplies home to work on with their families, and workbooks were brought to those in quarantine who were using their own art supplies to complete the projects. Over 70 mandalas were submitted and turned into a final workbook which will be available for download from the website for responders and families. A second multi-state "Mandala Challenge" is in the planning stages, starting with Virginia, Maryland and Washington, DC, where first responders will exchange mandalas, designing mandalas for others to create, and alternatively creating mandalas from the others' designs. In the co-design and creation of these mandalas, shared stories will be communicated, and these shared stories as expressed through the co-constructed mandala project will be digitized into an online art gallery.

\section{The Stress Ball Activity}

As a platform to relieve stress and enhance support and connection, the stress ball activity includes an integration of a participatory experience and a structured observational analysis. Participants were asked to crumple a piece of white copy paper in a tight ball, and to use art materials (e.g., markers, paints, oil pastels, chalk) to illustrate using color how their tensions and stress might look when most intensely experienced. They were then invited, when ready, to unfold the stress ball and carefully observe the flattened piece of paper. Dialogue focused on comparison between the 3-dimensional experience and the flattened two-dimensional paper, both of which held the same visual content, but yielded divergent perspectives when displayed differently, illustrating that what is observed may be different depending on its presentation even if it holds the same information. The unfolding process, analogous to change, may alter perspective, as participants observe the vast open white space available when that which was tightly held is released. Giving mindful attention to the nuances and details of the wound tension - to the unfolding - and to the new engagement with the opened work, allows new interpretations to emerge, such as deciding what to do now with the newly revealed open space. Participants often recognized that even if the stress ball was entirely covered in color, when they open it up, they see much more open empty space to work with and fill with what they choose. Contextual inquiry questions can focus the dialogue as "Contextual inquiry is about asking questions up close and in context, relying on observation, listening, and empathy to guide us toward a more intelligent, and therefore more effective, question" (Berger 2014, p. 97). Seeking out self-reflective questions of "what to do now with the new space I'm confronted with?" offers hope, inspiration, and control over decision-making about how to use time and about what emotions and behaviors should be let into that newly found space. The activity allowed for the power of kinesthetic stress release, where they could 'talk' about stress then engage in a physical experience to feel the difference between processes.

\section{A Virtual Family Summer Arts Camp}

Parental exposure to occupational distress can impact family members' sense of well-being. First responders are exposed to stress-inducing work-related incidents/events. Family environment should be examined as a focus of intervention for emotional regulation and relational support to mitigate the potential negative impact of occupational distress on first responder and family member well-being; and, yet there is no known current evidence-based interventions for first responders' children (Kishon et al. 2020).

This past Summer, Ashes2Art offered its inaugural First Responder Family Summer Arts Camp, with 52 participants comprised of a variety of emergency services personnel, including dispatchers, emergency room nurses, municipal and volunteer fire fighters, airport authority (fire fighters), paramedics and their families. The parents were urged to work with their children which cultivated familial connection between family members and within the responder family, such that two aspects of 'family' could be addressed. The camp focused on check-ins to reduce stress and to enhance responder family bonding. Art supply kits were distributed to all families and, after individual instruction, participants were invited to complete the art projects on their own, coming together as a community during four one-hour virtual video sessions over 8 weeks to share their created works. The work focused on "basic self-regulation skills" and "utilizing the resources around you", with a broad goal to diffuse stress so it does not become cumulative. Participants used tempura paints and made handkerchief batiks, blind contour line drawing self-portraits, outdoor mandalas, and cardboard sculptures out of packages that arrived during the pandemic. Virtual video sessions allowed families to come together to share and learn from each other, oriented around their art-making projects. The most important goal for the camp was to provide a platform for participants to experience (reexperience) the creative process and to freely explore, overcoming the burden of self-criticism or judgement. 


\section{Future Directions}

Next steps include designing a scientific study to empirically examine the emotional and physiological effects of mindfulness-focused arts programming for emergency services personnel and their families, using participants as their own controls over six-months. We hypothesize that direct and indirect regulation of the autonomic nervous system through modulating sensory input via mindfulnessfocused creative arts participation will reduce physiological distress and enhance first responders' confidence, control and coping during and after this COVID-19 pandemic. Enhanced regulation of the autonomic nervous system through sensory input monitoring and modulation may increase control over behavioral decisions. Control over behavioral decisions, behavioral regulation, is strengthened when a person is able to integrate mental, emotional, and physical (affective/bodily sensations) information (Hass-Cohen \& Findlay 2015). Increased Heart Rate Variability (HRV) is related to improved mental health and may be used as a proxy for underlying regulatory brain changes (Mather \& Thayer 2018); and, we hypothesize increased HRV and decreased daily stress levels will be associated with increased program participation.

Stimuli need to have personal connections and relate to the self to produce meaningful learned outcomes (Ann \& Hidi 2019), and this is accomplished in the hands-on artmaking experience. Digitization of participants' mandalas and other artwork can become "regulation anchors" that bring the person back to the original art-making experience, helping to ground and regulate when confronted with acute stress exposure, and potentially mitigating longer-term toxic effects of such exposures. An interactive screen interface that would allow the user to change the sensory characteristics of their digitized creative arts imagery-size, shape, color, texture - would enhance prolonged mindful engagement with the image. Over time, a digitized collection of these mindful regulation anchors could offer individuals real-time regulation tools, and repeated activation of the digitized imagery may train the brain and body to respond differently when presented with stressful situations that endanger well-being. In addition, participants would have the option to network into a creative arts community where their artwork resides in a digitized workbook along with those of other participants, creating a national creative community network by and for emergency services personnel. An inclusive community for connection, support, and compassionate dialogue through creative expression may facilitate a larger framework by which emergency services personnel can respond to and process the many challenges that crisis response brings to their work and family lives. Connecting to a larger support network encompassing their creative works and stories may result in shared learning experiences that leads to a sense of collective success.

\section{Conclusion}

Social workers actively engage in community work that brings wellness, hope and healing at multiple system levels. This work is most timely and needed right now. Helping first responders and their families manage the short- and longterm emotional toll from the work they do in responding to the COVID-19 crisis is paramount to the United States' successful recovery back to a well-functioning post-pandemic society. Enhancing health and mental health through mindfulness-focused creative arts engagement could be included as one part of routine self-care protocols for first responders and their families. We believe such programming allows first responders to be: Creatively Engaged - Socially Connected - Responder-Ready. A physically and mentally healthy first responder workforce is essential now and throughout postcrisis recovery.

Art-making offers strategies and tools that help tip towards the healthy and engaged end of the pandemic polarities, towards unity, connection, control and calm. Sensory support system interventions comprised of their own digitized artwork created out of the art-making experience, may preserve that calming effect by activating the regulated neurophysiological state originally experienced when creating art. Mobile technologies that enable individuals to become aware of when their brains and bodies are reacting to stress exposure provide their digitized mandala substitutions at that moment, along with immediate connections to their virtual and/or live peer support network, will help individuals gain awareness and control over their stress response, and offer a real-time mindful regulation strategy that they have created for themselves (Matto 2015; Matto \& Seshaiyer 2018; Matto et al. 2019).

It is critical to be able to detect acute stress and intervene early and effectively. First responders need to be "battleready" in their jobs, and the creative arts provide a safe platform to explore and manage the cumulative stress experienced, without causing detrimental vulnerability. Although didactic "wellness materials" are dispersed to first responders in great quantity, experiential wellness activities are often neglected at significant peril. Art-making offers the experiential and relational components that allow for the physiological release of embodied stress, and helps participants develop the skills and techniques they can draw on for ongoing self-care. In addition, a future opportunity is for Ashes2Art to provide a platform for community Art-making among emergency services personnel across the country through a national virtual space for creating and sharing 
artwork and self-care stories. Being able to digitally connect to a virtual network of other participants' artwork, regulation anchors and self-care stories, may offer an important community of support that is readily accessible when needed.

\section{References}

Ann, K., \& Hidi, S. E. (2019). Supporting the development of interest in the workplace In F. L. Oswald, T. S. Behrend \& L. L. Foster (Eds.), Workforce Readiness and the Future of Work (pp. 19-34). New York: Routledge.

Babouchkina, A., \& Robbins, S. J. (2015). Reducing negative mood through mandala creation: A randomized controlled trial. Art Therapy, 32(1), 34-39.

Baker, M. G., Peckham, T. K., \& Seixas, N. S. (2020). Estimating the burden of United States workers exposed to infection or disease: A key factor in containing risk of COVID-19 infection. PLoS One, 15(4), e0232452.

Barberian, M., Walker, M. S., \& Kaimal, G. (2019). 'Master my demons': Art therapy montage painting by active-duty military service members with traumatic brain injury and post-traumatic stress. Medical Humanities, 45, 353-360.

Beerse, M. E., Van Lith, T., Pickett, S. M., \& Stanwood, G. D. (2020). Biobehavioral utility of mindfulness-based art therapy: Neurobiological underpinnings and mental health impacts. Exp Biol Med (Maywood), 245(2), 122-130. https://doi.org/10.1177/15353 70219883634.

Berger, W. (2014). A more beautiful question: The power of inquiry to spark breakthrough ideas. Bloomsbury

Bracken-Scully, M., \& McGilloway, S. (2016). A qualitative exploration: Life after emergency services. International Journal of Emergency Services, 5(21), 158-173.

Campenni, C. E., \& Hartman, A. (2020). The effects of completing mandalas on mood, anxiety, and state mindfulness. Art Therapy, $37(1), 25-33$.

Carsley, D., \& Heath, N. L. (2019). Effectiveness of mindfulness-based coloring for university students' test anxiety. Journal of American College Health. https://doi.org/10.1080/07448481.2019.1583239.

Courtney, J. B., Lipsey, T., Braun, B., Henry, K., Nelson, T. L., \& Li, K. (2020). Using ecological momentary assessment to examine the effects of duty status on acute stress and tiredness in firefighters. Journal of Occupational and Environmental Medicine, 62(10), 859-870.

Denkova, E., Zanesco, A. P., Rogers, S. L., \& Jha, A. P. (2020). Is resilience trainable? An initial study comparing mindfulness and relaxation training in firefighters. Psychiatry Research, 285, 1-8. https://doi.org/10.1016/j.psychres.2020.112794.

DiJulio, B., Hamel, L., Munana, C., \& Brodie, M. (2018). Loneliness and social isolation in the United States, United Kingdom and Japan: An international survey. San Francisco, CA: Kaiser Family Foundation.

Fancourt D., \& Finn S. What is the evidence on the role of the arts in improving health and well-being? A scoping review. Copenhagen: WHO Regional Office for Europe; 2019 (Health Evidence Network (HEN) synthesis report 67).

Fergusson, D. M., Horwood, L. J., Boden, J. M., \& Mulder, R. T. (2014). Impact of a major disaster on the mental health of a wellstudied cohort. Journal of the American Medical Association Psychiatry, 71, 1025-1031. https://doi.org/10.1001/jamapsychi atry.2014.652 ([PubMed: 25028897]).

Garland, E. L., Farb, N. A., Goldin, P. R., \& Fredrickson, B. L. (2015). Mindfulness broadens awareness and builds eudaimonic meaning:
A process model of mindful positive emotion regulation. Psychological Inquiry, 26, 293-314.

Grebe, A. M. (2019). Healing our heroes: Creating an arts-based intervention. In J. Bopp, A. M. Grebe, \& J. H. Denny (Eds.). Advances in medical technologies and clinical practice (AMTCP). Healing through the arts for non-clinical practitioners. (pp. 22-43).

Hass-Cohen, N., \& Findlay, J. C. (2015). Art therapy \& the neuroscience of relationships, creativity, \& resiliency. WW Norton: New York, NY.

Henderson, P., Rosen, D., \& Mascaro, N. (2007). Empirical study on the healing nature of mandalas. Psychology of Aesthetics, Creativity, and the Arts, 1(3), 148-154. https://doi. org/10.1037/1931-3896.1.3.148.

Ho, A. H. Y., Tan-Ho, G., Ngo, T. A., Ong, G., Chong, P. H., Dignadice, D., \& Potash, J. (2019). A novel mindful-compassion art therapy (MCAT) for reducing burnout and promoting resilience for end-of-life care professionals: A waitlist RCT protocol. BMC Trials, 20, 406.

Huss, E., \& Sarid, O. (2014). Visually transforming artwork and guided imagery as a way to reduce work related stress: A quantitative pilot study. Arts Psychotherapy, 41, 409-412.

Kaimal, G., Ray, K., \& Muniz, J. (2016). Reduction in cortisol levels and participants' responses following art-making. Art Therapy: Journal of the American Art Therapy Association, 33(2), 74-80.

Kishon, R., Geronazzo-Alman, L., Teichman, M., Teichman, Y., Cheslack-Postava, K., Fan, B., et al. (2020). Parental occupational exposure is associated with their children's psychopathology. Journal of Occupational and Environmental Medicine, 62(11), 904-915.

Lee, S.-L. (2018). Why color mandalas? A study of anxiety-reducing mechanisms. Art Therapy, 35(1), 35-41.

Lowe, S. R., McGrath, J. A., Young, M. N., Kwok, R. K., Engel, L. S., Galea, S., \& Sandler, D. P. (2019). Cumulative disaster exposure and mental and physical health symptoms among a large sample of residents of the US gulf coast residents. Journal of Traumatic Stress, 32(2), 196-205.

Masterson-Duva, M., Haugen, P., Werth, A., Foster, A., Chassman, E., \& Breitbart, W. (2020). Adapting meaning-centered psychotherapy for World Trade Center psychotherapy for World Trade Center responders. Palliative and Supportive Care. https://doi. org/10.1017/S1478951520000061.

Mastandreal, S., Fagioli, S., \& Biasi, V. (2019). Art and psychological well-being: Linking the brain to the aesthetic emotion. Frontiers in Psychology. https://doi.org/10.3389/fpsyg.2019.00739.

Mather, M., \& Thayer, J. F. (2018). How heart rate variability affects emotional regulation brain networks. Current Opinion in Behavioral Sciences, 19, 98-104.

Matto, H. C. (2015). Bioehavioral response redirection: Innovations to activate personalized recovery cues and decrease relapse risk. Journal of Social Work Practice in the Addictions, 15, 450-453.

Matto, H. C., \& Seshaiyer, P. (2018). Harnessing the power of the recovering brain to promote recovery commitment and reduce relapse risk. Social Work in Journal of the Society for Social Work and Research, 9(2), 341-358.

Matto, H., Seshaiyer, P., Newcomb, A., Rothberg, S., \& Lopez-Piper, A. (2019). A novel mobile biobehavioral regulation system for personalized trauma recovery support. Patient Experience Journal, 6, 83-92.

Mayer, B. (2019). A review of the literature on community resilience and disaster recovery. Current Environmental Health Reports, 6, 167-173.

Miller, E. D. (2020). The COVID-19 pandemic crisis: The loss and trauma event of our time. Journal of Loss and Trauma, 25(6-7), $560-572$. 
Miller, A., \& Unruh, L. (2019). Individual and organizational influences of the professional quality of life of florida public safety personnel. International Journal of Emergency Services, 8(3), 221-235.

Ofei-Dodoo, S., Cleland-Leighton, A., Nilsen, K., Cloward, J. L., \& Casey, E. (2020). Impact of a mindfulness-based, workplace group yoga intervention on burnout, self-care, and compassion in health care professionals. Journal of Occupational and Environmental Medicine, 62(8), 581-587.

Rolland, J. S. (2020). COVID-19 pandemic: Applying a multisystemic lens. Family Process, 59(3), 922-936.

Segal-Engelchin, D., Achdut, N., Huss, E., \& Sarid, O. (2020). CB-Art interventions implemented with mental health professionalsworking in a sharedwar reality: Transforming negative images and enhancing coping resources. International Journal of Environmental Research and Public Health, 17(2287), 1-13.

Shapiro, S. L., Astin, J. A., Bishop, S. R., \& Cordova, M. (2005). Mindfulness-based stress reduction for health care professionals: Results from a randomized trial. International Journal of Stress Management, 12(2), 164-176.

Van Gordon, W., Shanin, E., \& Garcia-Campayo, J. (2017). The mandala of the present moment. Mindfulness, 8, 1720-1722.

Walsh, F. (2020). Loss and resilience in the time of COVID-19: Meaning making, hope, and transcendence. Family Process, 59(3), 898-911.
Williams, E., Dingle, G. A., Jetten, J., \& Rowan, C. (2019). Identification with arts-based groups improves mental wellbeing in adults with chronic mental health conditions. Journal of Applied Social Psychology, 49, 15-26.

Whitaker, A. (2016). Art thinking. New York: Harper Collins Publishers.

Yancy, C. W. (2020). COVID-19 and African-Americans. JAMA, 323(19), 1891-1892.

Publisher's Note Springer Nature remains neutral with regard to jurisdictional claims in published maps and institutional affiliations.

Dr. Holly C. Matto is an Associate Professor in the College of Health and Human Services Department of Social Work at George Mason University. She has conducted treatment intervention studies with diverse clinical populations, and enjoys working on multidisciplinary research teams designing mobile technologies for behavioral health symptom management in community settings. 